\title{
Quantitative assessment of increasing complexity
}

\author{
L.P. Csernai, S.F. Spinnangr, S. Velle \\ Institute of Physics and Technology, University of Bergen, Allegaten 55, 5007 Bergen, Norway
}

\begin{abstract}
We study the build up of complexity on the example of $1 \mathrm{~kg}$ matter in different forms. We start with the simplest example of ideal gases, and then continue with more complex chemical, biological, life, social and technical structures. We assess the complexity of these systems quantitatively, based on their entropy. We present a method to attribute the same entropy to known physical systems and to complex organic molecules, up to a DNA molecule. The important steps in this program and the basic obstacles are discussed.
\end{abstract}

\section{Introduction}

The problem of development is longstanding in humanity. It became quantitative with the development of statistical physics and quantum physics. The first theoretical step was done by Boltzmann, who laid down the basis of microscopic quantitative treatment of entropy both for equilibrated and out of equilibrium systems. In the H-theorem he showed that closed systems in equilibrium maximize their entropy, and all microscopic interactions drive the system towards this equilibrium. This macro state is the most probable one the system can reach.

Consequently, a less probable, non-equilibrium state can be formed only if the system is not closed and can exchange entropy with the surrounding, so that its own entropy decreases. Under stationary conditions, constant pressure and temperature, spontaneous chemical reactions do not lead to more complex systems.

These ideas were discussed already by E. Schrödinger in 1943, in his book: "What is life?" [1]. He described that life forms are highly complex systems of a high level of "order", that can develop from disordered systems, or more probably from ordered systems of a somewhat lower level of order. Our ultimate aim is to show at what cost sustainable development is possible, what the direction of sustainable development is, and which processes are working towards such development. We aim to quantify the LEVEL and the RATE of the development quantitatively.

Schrödinger described the problem and the concepts in a genius way, but he could not give quantitative information at that time. We know much more about biological structures and life forms today, so it is possible to discuss these problems quantitatively.

In this work we introduce the method to calculate the entropy of a well defined amount of matter, irrespective of what form of matter we are discussing. This is because the development on the Earth happens with a constant amount of matter, but the forms of matter may change due to nuclear, chemical, biological, technological, intellectual and societal reactions and changes. Thus, we introduce a unit of $\mathbf{1} \mathbf{~ k g}$ for our discussion. (Although, for intellectual and societal changes and structures this choice of unit is too extreme in this moment.) The human intellect is the limit where the material form, the nervous system, and the intellectual information content are at the boundary of our knowledge. At this time this program can be performed up to simple structures of actions and simple vegetative nervous systems. For transparency and the illustration of the program we start from the simplest (and highest entropy) 
systems. We intend to provide a simple and sometimes even simplified presentation of the entropy calculations and the corresponding degrees of freedom of materials.

The second important new aspect we introduce, is to achieve the possibility of comparing different forms of matter with a uniform definition of entropy. In the present literature, in mathematics, information theory, linguistics, etc. many definitions of entropy exist. The most widely known, after the material entropy in statistical physics, is the "Shannon entropy" [2]. We remind the reader that this definition was already used by Boltzmann in the H-theorem for non-equilibrium systems, and this makes it possible to attribute the same physical dimension to both entropies.

A third aspect we have to utilize is the quantization of the phase space entropy by the volume of the phase-space cell. This is an important step in unifying the entropy evaluation and it is not obvious how to perform this for highly complex systems.

The last step is to select the physically realized system configuration(s) from all possible ones with the same degree of complexity and degrees of freedom. In highly complex systems this step is also nontrivial, and for societal or intellectual systems it is even debatable which systems are realized or realizable.

We introduce this system in a series of examples, from the most simple elementary ones up to the most complex ones, which can still be discussed on a quantitative basis. These last examples are from biology, but our aim is not to contribute to quantitative biology, but rather to demonstrate our procedure, how to quantitatively assess the sustainable development following Schrödinger's original ideas.

\begin{tabular}{crrrr}
\hline \hline Material & $A_{P}$ & $\begin{array}{r}N_{P} \\
(\mathrm{~mol} / \mathrm{kg})\end{array}$ & $\sigma_{P}$ & $\begin{array}{r}S_{1 k g} \\
(\mathrm{~J} / \mathrm{K})\end{array}$ \\
\hline $\mathrm{H}_{2}$ & 2 & 496.046 & 14.146 & 58344.0 \\
$\mathrm{He}$ & 4 & 248.023 & 15.186 & 31316.1 \\
$\mathrm{H}_{2} \mathrm{O}$ & 18 & 55.116 & 17.442 & 7993.0 \\
$\mathrm{H}_{2} \mathrm{O}^{*}$ & 18 & 55.116 & 17.988 & 8243.0 \\
$\mathrm{Rn}_{\mathrm{N}}$ & 222 & 4.469 & 21.211 & 788.1 \\
$\mathrm{WF}_{6}$ & 298 & 3.329 & 21.652 & 599.3 \\
$\mathrm{UF}$ & 352 & 2.818 & 21.902 & 513.2 \\
$\mathrm{C}_{6} 0$ & 720 & 1.378 & 22.975 & 263.2 \\
\hline
\end{tabular}

Table 1. Thermodynamical parameters of $1 \mathrm{~kg}$ material in different forms approximated as ideal gases, depending on their mass numbers, $A_{P}$, different mol-numbers of particles, $N_{P}$, and this number is decreasing with increasing $A_{P}$. The specific entropy of the composite particles is indicated by the dimensionless $\sigma_{P} \quad\left(\hbar, c, k_{B}=1\right)$. The total entropy of the material at the used $T=300^{\circ} \mathrm{K}$ and $p=1$ bar, is also decreasing with increasing complexity, i.e. increasing $A_{P}$. (For $\mathrm{H}_{2} \mathrm{O}^{*}$ the temperature is taken to be $T=100^{\circ} \mathrm{C}=373.15^{\circ} \mathrm{K}$.)

\section{Elementary Entropy Evaluations for Unit Amount of Matter}

In this section we introduce the first steps of our program, with the basic units and definitions using the most simple, generally known systems. For those who are well familiar with statistical physics the sections up to Section $4(\operatorname{ref}\{S 3\})$ or $5(\operatorname{ref}\{S 4\})$ can be skimmed through. Here the importance of quantization and the unified treatment of Gibbs and Shannon entropy are essential.

We can start with the example of more and more complex chemical structures. For example with dilute gases, approximated first as ideal gases.

We take a series of gases, $\mathrm{H}_{2}, \mathrm{He}, \mathrm{H}_{2} \mathrm{O}, \mathrm{Rn}, \mathrm{WF}_{6}, U F_{6}$ and $\mathrm{C}_{60}$, with increasing molecular weight or "Particle mass Number", $A_{P}$. We take $1 \mathrm{~kg}$ of material, so the number of particles, $N_{P}$, in this amount of matter will decrease with increasing mass number, $A_{P}$. We choose that the gas is at standard atmospheric pressure, i.e. at $P=1 \mathrm{~atm}$, and $T=300^{\circ} \mathrm{K}$ temperature.

Using the ideal gas approximation

$$
S_{1 \mathrm{~kg}}=N_{P} k_{B}\left[\frac{5}{2}+\ln \left(\frac{\left(2 \pi m_{P} c^{2} k_{B} T\right)^{3 / 2}}{n_{P}(2 \pi \hbar c)^{3}}\right)\right],
$$


where $k_{B}$ is Boltzmann constant $\left(k_{B}=1.38064852 \cdot 10^{-23} \mathrm{~J} / \mathrm{K}\right), m_{P}$ is the particle mass, (we take it as $m_{P}=A_{P} m_{N u c l}$. in terms of the nucleon mass, $\left.m_{N u c l}\right)$ and $n_{P}$ is the particle density $\left(n_{P}=A v /\left(V_{I . G}\right)\right.$ in terms of the Avogadro number, $A v$. The molar volume of an ideal gas is, $V_{I . G}$. at STP, standard pressure and standard temperature of $\left.T=273.15^{\circ} \mathrm{K}\right)$. The moderately increasing dimensionless specific entropy per particle, $\sigma_{P}=S /\left(k_{B} N_{P}\right)$, is also shown. See Table 1 In the table all values were calculated in the ideal gas approximation and for $\mathrm{T}=300^{\circ} \mathrm{K}$, except the water, $\mathrm{H}_{2} \mathrm{O}$, which is at boiling temperature, $T=373.15^{\circ} \mathrm{K}$.

Noble gases, $\mathrm{He}, \mathrm{Rn}$, and also small molecules, can be well approximated as ideal gases, their vibrational degree of freedom carries negligible energy. Under standard conditions the heaviest couple of materials are not gases and their interactions and internal degrees of freedom should also be taken into account. Thus, for water vapour and for the heavy gases the ideal gas approximation underestimates the entropy of the material.

Nevertheless, even for ideal gases the entropy is not behaving the same way as the particle number. This can be seen well in Fig. 1. Let us take the values of $N_{P}$ and $S_{P}(1 \mathrm{~kg})$ for $H_{2}$ as standard unity, and see how $N_{P}$ and $S_{P}(1 \mathrm{~kg})$ change with increasing $A_{P}$. While $N_{P}$ decreases according to the $N_{P} A_{P}=$ const. constraint (and therefore $\left.N_{P} \propto A_{P}^{-1}\right)$, the entropy, $S_{P}(1 \mathrm{~kg})$, decreases less due to the additional contribution of the log term in the entropy expression $\propto \ln \left(m_{P} / n_{P}\right)$. Consequently the entropy decreases as $S \propto A_{P}^{-0.9}$.

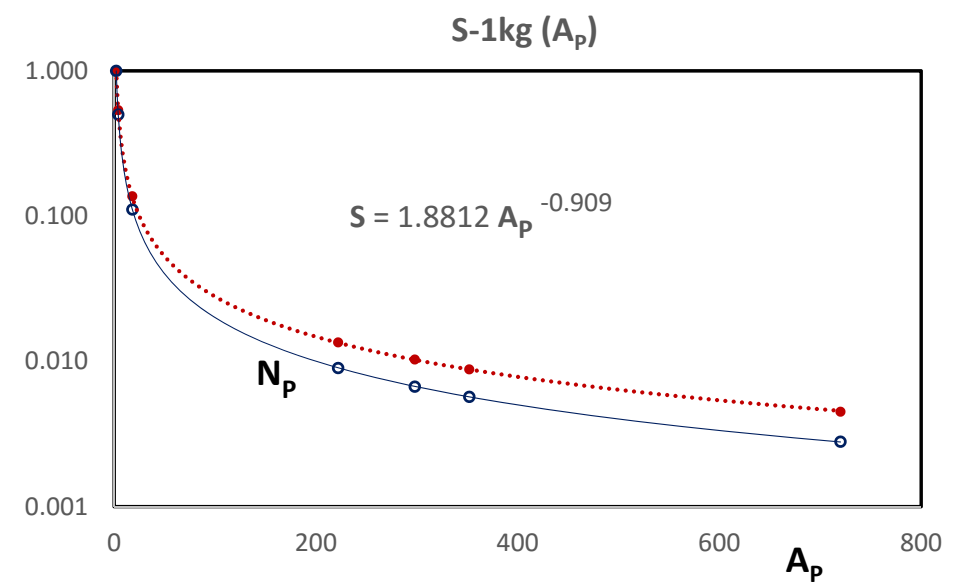

Figure 1. (color online) The mass number, $A_{P}$, dependence of the relative entropy of $1 \mathrm{~kg}$ material, $S$, compared to the number of these particles, $N_{P}$. The relative entropy, $S$, as well as the particle number, $N_{P}$, of $1 \mathrm{~kg} \mathrm{H}_{2}$ is taken to be unity for the comparison. The entropy decreases slower than the decrease of the particle number. This means that the entropy per particle is increasing.

This change is shown if Fig. 2, where again the ratio $\sigma_{P}=S /\left(k_{B} N_{P}\right)$ for $H_{2}$ is taken to be unity. The increasing relative entropy exceeds $S /\left(k_{B} N_{P}\right)=1.6$ for $C_{60}$ but it has a saturating tendency. In this ideal gas approximation the ratio is not expected to exceed two.

For larger, more complex molecules the number of degrees of freedom increases, and specific molecular configuration has less entropy than the completely random ideal gas.

Interacting materials can form other liquid or solid phases, which have more constraints, compared to the increasing number of degrees of freedom. This decreases the entropy of a given liquid or solid further. We will illustrate this on the example of water.

As in liquids there is still considerable room for random configurations where entropy is decreasing less than that of the solids, where the level of "order" [1] is higher.

Schrödinger's considerations were extended to lifeforms of matter [1], and similar comparative studies were used for comparing the change of the entropy content of different species during their lifespan [3], as well as the rate of change of the entropy during the life of different species. The initial development phase leads to a considerable decrease of the entropy of the matter incorporated into the living species (build up of neg-entropy), then the entropy is increasing again in the second stage of the life of the species. The rate of these changes is connected to the energy exchange with the environment (or metabolism) and this then determines the lifespan of a species. 


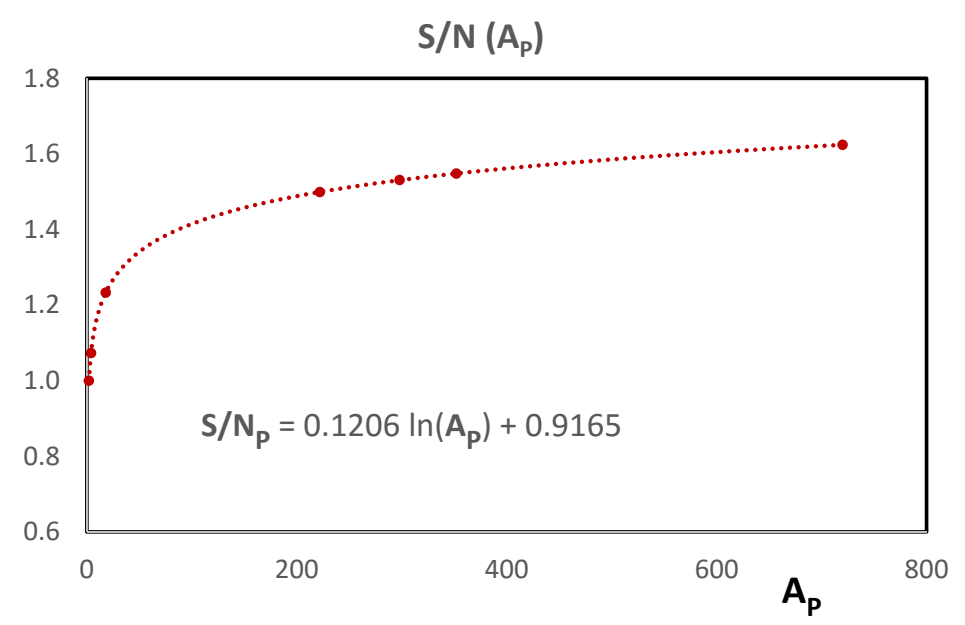

Figure 2. (color online) The change of the relative ratio of entropy versus particle number, $S / N_{P}$ as a function of the mass number of a given particle species, $A_{P}$. For this comparison the $S / N_{P}$ for $H_{2}$ is taken to be unity.

Interestingly Schrödinger discusses two ways on how to achieve a high level of order, (i.e. smaller entropy). High level of order can be built up from dis-ordered materials as well as from an already ordered material with achieving a higher level of order. This second way is obviously easier and preferable.

Interestingly the same fundamental ideas can be extended to the sustainable development of the Earth, and this has also been done for a while, using the principles of statistical physics [4]. The consequences of these more fundamental and more quantitative considerations are interesting. The popular folklore considers some selected energetic processes as sustainable, while others are not. Recently some energy resources are declared "renewable" others are not. In this respect the use of "bio-fuels" is quite problematic, because if we burn or destroy highly complex, biological materials, this may lead to decreasing "order" in Schrödinger's sense, which is not contributing to sustainable development. The same issue may arise if photovoltaic production sites occupy large agricultural territories, which is not taken into consideration.

Therefore our aim is to demonstrate the connection between sustainable development, energy and entropy exchange in a quantitative way, as far as possible.

Furthermore, we want to extend these considerations beyond different life-forms of matter, to technical and social development. In this last point, quantitative energetic and entropy aspects are probably beyond our present knowledge, but one can still estimate which direction of changes certain social actions can or will cause.

\section{Network or topological entropy}

We can continue the previous simplified study by considering different hypothetical ideal gases constructed from nucleons like $\mathrm{H}_{1}, \mathrm{H}_{2}, \mathrm{H}_{3}, \mathrm{H}_{4}$. If we consider these as different ideal gases without taking into account the type of binding, then we end up with the result in the previous section. Let us neglect the physical features of a binding, such as its energy or extra degrees of motion, and only consider the possible topologies of the binding. If we take the molecule $\mathrm{H}_{2}$, then we have a link between two nucleons. For this molecule we can only insert a link one way to make a cluster, so the existence of the link does not contribute to extra energy or entropy (because we neglected the small rotational or vibrational energies).

In both cases $\left(H_{1}\right.$ and $H_{2}$ ) there is only one configuration for the molecule, i.e. $i=1$ and $p_{1}=1$, and the sum of all allowed configuration states is, $N$, is $N=1$. Thus, the topological Shannon entropy of these molecules is

$$
H(X)=-p_{1} \ln p_{1}=0 \text {. }
$$


If $N$ would be $N>1$, then the most random configuration would be $p_{i}=1 / N$ for each $i$ and so for this configuration the Shannon entropy is

$$
H(X)=-\sum_{i=1}^{N} p_{i} \ln p_{i}=-N \frac{1}{N} \ln \frac{1}{N}=\ln N=H_{\max }
$$

Let us now consider a (hypothetical) $H_{3}$-molecule. This can be formed by inserting (2) links or (3) links! Thus, we can have two characteristic structures for 3 nucleons. Two links, (2), can be inserted 3 ways: $12 \& 23$ or $13 \& 23$ or $13 \& 12$ for identical but distinguishable nucleons. Three links, (3), can be inserted only one way, 123, for identical particles. The two link configurations can be obtained from the three link ones by cutting one link and this can be done in three ways. This is altogether 4 configurations, with (3) being $i=1$ and (2) being $i=2$, then $p_{1}=1 / 4$ and $p_{2}=3 / 4$. The Shannon entropy of a system $X$ with all possible configurations of the $H_{3}$ molecule is then

$$
H(X)=-\left[\frac{1}{4} \ln \frac{1}{4}+\frac{3}{4} \ln \frac{3}{4}\right]=0.5623 .
$$

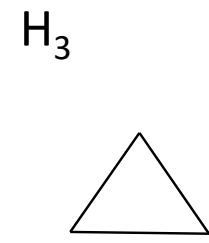

(3)

Figure 3. The topological configurations of hypothetical $H_{3}$ molecules, according to the number of links in a given configuration, (3) or (2). These configurations can be formed in $N_{i}=1,3$ ways respectively.

If we consider a (hypothetical) $H_{4}$-molecule, then the maximum number of connections is 6 and the minimum number (keeping still a bound cluster) is 3 . A molecule with 5 bounds can be obtained by cutting one of the 6 bounds, and this can be done 6 ways. A molecule with 4 bounds can be obtained by cutting one more bound, as the lines are indistinguishable we do not take the order of which we remove the lines into account. This can then be done in $6 * 5 / 2$, so altogether this gives 15 configurations, but these will have two different topological configurations:

(4A) 1 way with each node having 2 links, i.e. in tot. $N_{(4 A)}=3 * 1$ ways and

(4B) 4 ways with nodes having $1,2,2 \& 3$ links, i.e. in tot. $N_{(4 B)}=3 * 4$ ways.

Then with a further cut $(4 \mathrm{~A})$ leads to a linear chain with 3 links (3A) which can be obtained 4 ways, i.e. in tot. $3 * 1 * 4 / 3$ ways. The (4B) configuration leads to the (3A) chain in 2 ways by cutting one of the links at the 3-link node, in tot. $3 * 4 * 2 / 3$ ways. Thus the (3A) configuration can be reached in total $N_{(3 A)}=3 * 4 * 3 / 3$ ways.

There is a further 3 link configuration which can be generated from (4B) by cutting the link between the two 2-link nodes, this can only be done one way, i.e. $N_{(3 B)}=3 * 4 * 1 / 3$ ways in total.

Thus the (6) and (5) link structures have only one type of topological configuration, although with different topological formation probabilities, the (3) and (4) link configurations have two types of topological configurations, A and $\mathrm{B}$, and these have different topological formation probabilities. The sum of all configurations is $N=\sum_{i} N_{i}=38$ and so, $p_{i}=N_{i} / N=0.0263,0.1579,0.0789,0.3158,0.3158,0.1053$.

If all these configurations are realized, with the above probabilities then the Shannon entropy of the system is

$$
H(X)=-\sum_{i=1}^{N} p_{i} \ln p_{i}=-\sum_{i=1}^{6} \frac{N_{i}}{N} \ln \frac{N_{i}}{N}=1.5526 .
$$

This entropy is a sum of all six possible configurations. The entropy of these six subsets is additive so we can also calculate the entropy of a given configuration or of a subset of configurations. Thus for the configuration $\mathrm{X}=(4 \mathrm{~A})$, we 


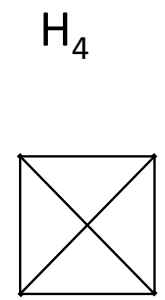

(6)

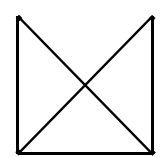

(5)

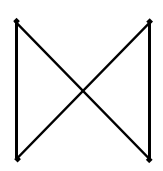

(4A)

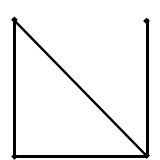

(4B)

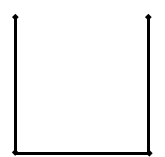

(3A)

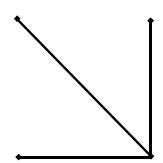

(3B)

Figure 4. The topological configurations of hypothetical $H_{4}$ molecules, according to the number of links in a given configuration, (6), (5), (4A), (4B), (3A), (3B). These configurations can be formed in $N_{i}=1,6,3,12,12,4$ ways respectively.

obtain

$$
H(4 A)=0.3662 \text {. }
$$

Thus the connection or network topology should also be taken into account. We can consider this on the hypothetical example of $\mathrm{H}_{1}, \mathrm{H}_{2}, \mathrm{H}_{3}, \mathrm{H}_{4}$ gases, with and without taking into account the topological entropy.

In real physical situations, not all (hypothetical) configurations are realized. Furthermore, the links also have energies, so the experimental probabilities are not exactly the same as the topological estimates. Nevertheless, this topological example is able to give guidance on how to take topological (or network) structures into account in entropy estimates.

In the case of usual (Shannon) entropy estimates, the normalization is not the same as the physical one, but it is perfectly sufficient for comparative studies of these types of structural entropies.

Now the question arises, how do we add this configuration entropy to the expression in eq. (1)? For this purpose we convert both definitions into dimensionless form. The configuration entropy, i.e. the Shannon entropy is already in dimensionless form. Boltzmann's original definition for any (non-equilibrium) system has also the same structure. The definition for one particle

$$
H(X)=\sigma_{P}=\frac{S}{k_{B} N_{P}}=-\sum_{i=1}^{N} p_{i} \ln p_{i},
$$

may define both the sum for the probabilities of all configurations, $p_{i}^{c}$, or the sum for all probabilities to be in a phase space volume element, $p_{i}^{p}$, of size $(2 \pi \hbar)^{3}$. This in this latter case is

$$
p_{i}^{p}=(2 \pi \hbar)^{3} f(x, p),
$$

where $f(x, p)$ is the phase space distribution function normalized for one particle. In this latter case $\sigma_{P}$ is the dimensionless specific entropy for one particle (or molecule), which can be obtained from the physical entropy $S$ as given by eq. (7).

For a relativistic gas, the entropy density, using Boltzmann's definition for any equilibrium or non-equilibrium phase space distribution, $f(x, p)$, is [5]

$$
s(x)=-\int \frac{d^{3} p}{p^{0}} p^{\mu} u_{\mu} f(x, p)\left[\ln \left((2 \pi \hbar)^{3} f(x, p)\right)-1\right],
$$

where $p^{\mu} u_{\mu}$ is the frame invariant relativistic expression for the local energy density. The last term, -1 , ensures the appropriate entropy constant for joining smoothly the low temperature quantum statistical limit in case of Boltzmann 
statistic 11

If we reach an equilibrium, we have a stationary solution of the Boltzmann Transport Equation, e.g. the Jüttner distribution, $f(x, p)$, and the entropy becomes

$$
s(x)=-\int d^{3} p f(x, p)\left[\frac{\mu}{T}-\frac{\varepsilon}{T}-1\right] .
$$

Here $\mu$ is the chemical potential, $T$ is the temperature and $\varepsilon$ is the specific energy, the energy for one particle. Notice that in the relativistic theory both $\mu$ and $\varepsilon$ includes the rest mass of the particle, but in the entropy expression these terms cancel each other. In the non-relativistic limit for the Boltzmann distribution this leads to the entropy expression of eq. (1).

If the phase space distribution is normalized to $n_{P}(x)$, i.e.

$$
n_{P}(x)=\int d^{3} p f(x, p),
$$

then the entropy for one particle can be obtained for the Boltzmann statistics as

$$
\sigma_{P}^{p h . s .}=\frac{s}{k_{B} n_{P}}=-\sum_{i \in \text { ph.s. }} p_{i}^{p}\left[\ln p_{i}^{p}-1\right]
$$

where $p_{i}^{p}=(2 \pi \hbar)^{3} f(x, p)$, the probability to be in a phase space cell, $i$, should be calculated for one particle, i.e.

$$
\int d^{3} x n_{P}(x)=1 .
$$

Still the entropy, the distribution function and $p_{i}^{p}$ depend on the particle density, $n_{P}$.

Thus in conclusion the single particle entropies should be additive in a configuration

$$
\sigma_{P}=\quad \sigma_{P}^{\text {conf. }}+\sigma_{P}^{\text {ph.s. }} \quad=-\sum_{i \in \text { conf. }} p_{i}^{c} \ln p_{i}^{c}-\sum_{i \in \text { ph.s. }} p_{i}^{p}\left[\ln p_{i}^{p}-1\right],
$$

where $p_{i}^{c}$, is the probability to have a configuration state, $i$. In conclusion the increase of the degrees of freedom due to the different configurations leads to an increase in the single particle entropy. This increase is very small if the number of possible configurations, $N=\sum_{i} N_{i}$ is large, but the number of a realized configurations, $N_{i}$, is much smaller, $N_{i} \ll N$.

In the above discussed estimate we only considered differences in topological configuration among identical particles. In real situations the complexity may increase due to: direction dependence of the links, different constituents, different (energetic) weights of the links, dynamical freedom of the length or angle of the link, etc.

In Table 2 the hypothetical ideal gas particles, $H_{1}$ and $H_{2}$, have no option for different configurations. For $H_{3}$, the specific entropy, $\sigma_{P}$, increased by a relatively small amount of 0.347 and 0.216 for the topological configurations $H_{3}^{(3)}$ and $H_{3}^{(2)}$ respectively. The entropy of the different $H_{4}$ configurations is increased by small values of configuration entropy between 0.096 and 0.364 . The increase in configuration entropy is very small for configurations with small probability, $p_{i}$, which may occur for complex systems with a large number of possible configurations where only a few are realized in a realized sample of configurations.

The configuration entropy may be much larger for more involved structures, where the number of configurations are comparable or may even exceed the number of particles. Also, an important question to ask is how many of these configurations can be realized, and how many are actually present in a given sample we discuss.

\footnotetext{
${ }^{1}$ For Fermi-Dirac or Bose-Einstein distributions the additional term, -1, does not appear, but at the same time the calculation of the probabilities of the phase space cell occupations is more involved.
} 


\begin{tabular}{crrrr}
\hline \hline Material & $A_{P}$ & $\begin{array}{r}N_{P} \\
(\mathrm{~mol})\end{array}$ & $\sigma_{P}$ & $\begin{array}{r}S_{1 k g} \\
(\mathrm{~J} / \mathrm{K})\end{array}$ \\
\hline$H_{1}$ & 1 & 992.092 & 13.106 & 108111.7 \\
$H_{2}$ & 2 & 496.046 & 14.146 & 58344.0 \\
$H_{3}$ & 3 & 330.697 & 14.754 & 40568.3 \\
$H_{3}^{(3)}$ & 3 & 330.697 & 15.101 & 41521.2 \\
$H_{3}^{(2)}$ & 3 & 330.697 & 14.970 & 41161.6 \\
$H_{4}$ & 4 & 248.023 & 15.186 & 31316.1 \\
$H_{4}^{(6)}$ & 4 & 248.023 & 15.282 & 31513.5 \\
$H_{4}^{(5)}$ & 4 & 248.023 & 15.477 & 31917.1 \\
$H_{4}^{(4 A)}$ & 4 & 248.023 & 15.386 & 31729.5 \\
$H_{4}^{(4 B)}$ & 4 & 248.023 & 15.550 & 32066.8 \\
$H_{4}^{(3 A)}$ & 4 & 248.023 & 15.550 & 32066.8 \\
$H_{4}^{(3 B)}$ & 4 & 248.023 & 15.423 & 31804.8 \\
\hline
\end{tabular}

Table 2. Entropies of a single composite particle and of $1 \mathrm{~kg}$ material in different topological configurations for hypothetical $H_{1}, H_{2}, H_{3}$ and $H_{4}$, molecules approximated as ideal gases, depending on the mass numbers, $A_{P}$, of the nucleons in the molecule and the configuration where it is indicated. $1 \mathrm{~kg}$ material contains different mol-numbers of particles, $N_{P}$, and this number is decreasing with increasing $A_{P}$. The specific entropy of the composite particles is indicated by the dimensionless $\left(\hbar, c, k_{B}=1\right) \sigma_{P}$. The total entropy of the $1 \mathrm{~kg}$ material at STP is also decreasing with increasing complexity, i.e. increasing $A_{P}$.

\section{Entropy of Phases of Physical Systems}

Physical systems may have a variety of configurations, different (i.e. nonidentical) constituents, and different physical degrees of freedom, such as vibration, rotation, etc., in addition to the phase space occupancy. Because of this, their entropy may well exceed the entropy based on the ideal gas approximation. E.g. $1 \mathrm{~kg}$ ideal gas with mass number $18\left(\mathrm{H}_{2} \mathrm{O}\right)$ has an entropy of $S_{1 \mathrm{~kg}}=8243.0 \mathrm{~J} / \mathrm{K}$, at the boiling point, while the physical value is about 10495 $\mathrm{J} / \mathrm{K}$. This can be explained by the different types of constituents, $H$ and $O$, the configuration and other dynamical degrees of freedom and types of interactions.

If we add the topological configuration entropy, $\sigma_{P}^{\text {conf. }}$, to the ideal gas estimate of the $\mathrm{H}_{2} \mathrm{O}^{*}$ molecule (at $T=$ $100^{\circ} \mathrm{C}$ ), we get a larger value. Here we have two possibilities, the two Hydrogen atoms can be identical (or not). In these two cases we have 3 (or 4) possible topological configurations, see Fig. 6. The specific topological configuration entropies, $\sigma_{P}^{\text {conf. }}$, can be calculated as in section 3 . Here we assume equal probability for each topological configuration. There are four possible cases: either all possible configurations are realized or only a single one, and either the $H$ atoms are identical ( $N=4$ possible cases) or not ( $N=3$ possible cases). Then, the entropy can be calculated as:

$$
\sigma_{P}^{\text {conf. }}=-\sum_{i=1}^{N} p_{i} \ln p_{i}=\ln N \quad \text { or } \quad \sigma_{P}^{c o n f .}=-p_{i} \ln p_{i},
$$

for all configurations or a single one respectively. These additive topological configuration entropies are given in Table 3. together with the total entropy values where the entropy from the phase space occupation, $\sigma_{P}^{\text {ph.s. }}=17.988$ and for the topological configuration are added up.

If all three (or four) configurations would be realized with the same probability then we get a larger final specific entropy, $\sigma_{P}=\sigma_{P}^{\text {conf. }}+\sigma_{P}^{\text {ph.s. }}=19.087(19.374)$ and the corresponding entropy for $1 \mathrm{~kg}$ material will become $S_{1 \mathrm{~kg}}=$ $8746.6(8878.3) \mathrm{J} / \mathrm{K}$. If only one configuration is realized from the three (or four) possible ones, then the final entropies are smaller. For the specific entropy $\sigma_{P}=18.354(18.335)$ while for $1 \mathrm{~kg}$ of material $S_{1 \mathrm{~kg}}=8410.9$ (8402.1) $\mathrm{J} / \mathrm{K}$. These last smaller values indicate that the larger information content (i.e. that only one state is realizable from the possible ones) decreases the entropy of the given material.

We see that the estimate that includes the addition of topological entropy moved the estimated entropy of the system closer to the experimentally observed value. Obviously, we did not reach the experimental value as several 


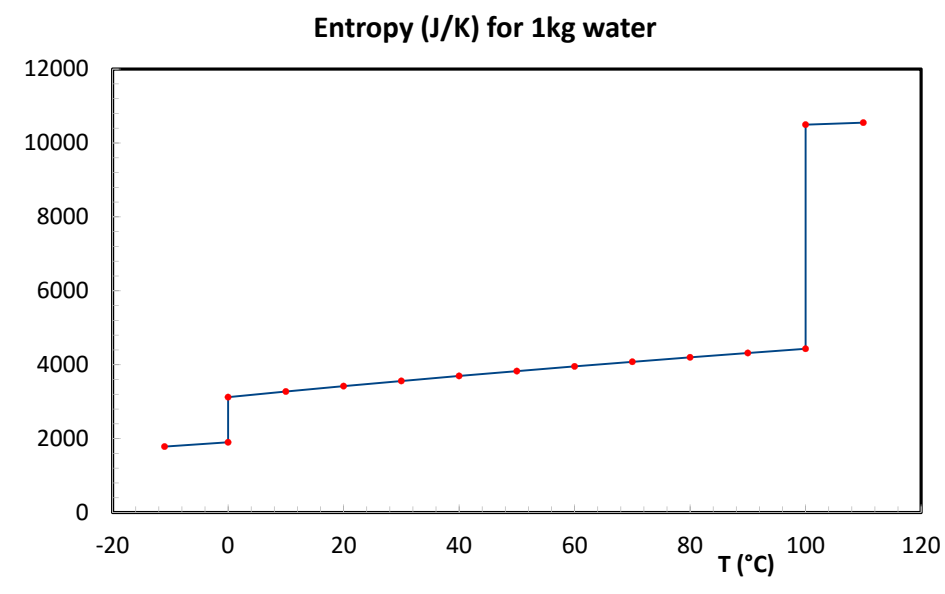

Figure 5. (color online) Entropy of $1 \mathrm{~kg}$ of water at different temperatures, with all three phases, ice, water and vapour, with two phase transitions at 0 and $100^{\circ} \mathrm{C}$. The reference point is taken at $T=100^{\circ} \mathrm{C}$ liquid water, with entropy of $S_{1 \mathrm{~kg}}=4430.01 \mathrm{~J} / \mathrm{K}$, where the phase transition to vapour with a latent heat of $\Delta S=6065.55 \mathrm{~J} / \mathrm{K}$, leads to a vapour entropy of $S_{1 \mathrm{~kg}}=10495.56 \mathrm{~J} / \mathrm{K}$. Using the water heat capacities we reach the phase transition between water and ice with water entropy of $S_{1 \mathrm{~kg}}=3122.92 \mathrm{~J} / \mathrm{K}$ and ice entropy of $S_{1 \mathrm{~kg}}=1900.15 \mathrm{~J} / \mathrm{K}$.

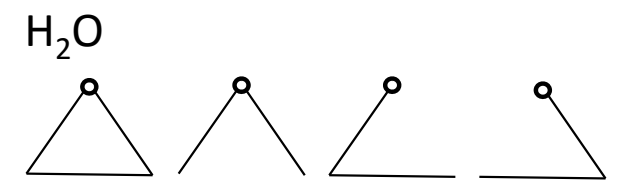

Figure 6. The topological configurations of a $\mathrm{H}_{2} \mathrm{O}$ molecule. The circle indicates the Oxygen atom. If the two $\mathrm{H}$ atoms are identical then the last two configurations are identical also.

other degrees of freedom (angles, lengths, etc.) were not taken into account, nor the energetic configuration of the water molecule in the vapour.

The exact absolute values of ice are less well known, because the ice has several different configuration structures and it is difficult to make measurements down to $0^{\circ} \mathrm{K}$ temperatures. The theoretical calculation of complex energetic structures is difficult and the theoretical calculations of structural changes of a phase transition is even more so.

At the phase transitions the heat capacity has a pole, which is again problematic to calculate, so the exact "calculation" of absolute entropies is already difficult for relatively simple molecules with a phase transition.

Nevertheless, we attempt to estimate the absolute entropy values of more complex molecules also, where experimental entropy measurements do not exist. There are no fundamental reasons to prevent us from estimating the absolute entropies of highly complex molecules.

For the fundamental determination of absolute entropy values it is important that (i) we quantized the phase space cells with a well defined physical value, and (ii) we connected the entropy to a well defined amount of matter and its energy.

The role of configuration entropy is in principle clear. The 3 atoms of water belong to the above discussed configurations, but the weight of the different probabilities for these configurations are of course different because the connections carry energy, which depends on the "angle" of the two $\mathrm{H}$ atoms in the $\mathrm{H}_{2} \mathrm{O}$ molecule. Or more precisely, these depend on the characteristic angle of the quantum mechanical wave function of the molecule. In different model calculations this angle varies between $104.52^{\circ}$ and $109.5^{\circ}$, and it may also be dependent on the density and temperature of the material. 


\begin{tabular}{crrrr}
\hline \hline & All 4 & All 3 & One of 4 & One of 3 \\
\hline$\sigma_{P}^{\text {conf. }}$ & 1.386 & 1.099 & 0.347 & $\mathbf{0 . 3 6 6}$ \\
$\sigma_{P}$ & 19.374 & 19.087 & 18.335 & $\mathbf{1 8 . 3 5 4}$ \\
\hline$S_{1 k g}^{\text {conf. }(\mathrm{J} / \mathrm{K})}$ & 635.1 & 503.63 & 159.02 & $\mathbf{1 6 7 . 7}$ \\
$S_{1 \mathrm{~kg}}(\mathrm{~J} / \mathrm{K})$ & 8878.3 & 8746.8 & 8402.2 & $\mathbf{8 4 1 0 . 9}$ \\
\hline
\end{tabular}

Table 3. Topological configuration entropy and total entropy of $100^{\circ} \mathrm{C}$ water vapor. The realistic physical case is in the last column.

This feature indicates that the probabilities of the real physical configurations may not be the same as in the oversimplified configuration estimates presented in the previous section. Some configurations can have a highly suppressed probability, or might not be realized at all in nature. E.g. for water the probability of first configuration in Fig. 6, is probably much smaller (based on the wave-function) than in the above topological estimate.

We will see that in more complex structures, there are possible configurations which do not appear in nature at all. To find out which configurations are realized and with what probability, is a much more difficult question than just counting up the number of possible configurations.

The ratio of configuration entropy to kinetic entropy: In Table 1 one we could see that the kinetic entropy increases with increasing particle mass, but this increase is rather slow. Furthermore, this table was calculated with the ideal gas approximation i.e. like the water vapour phase of water. If we consider liquid water at a lower temperature, e.g. room temperature, the entropy of the system is reduced by about 60\%, as shown in Fig. 6 While for water vapour the ratio of kinetic entropy to configuration entropy is roughly $20: 1$, for liquid water at room temperature it is only about 5:1 ! With more complex molecules the weight of configuration entropy increases further and it may overcome the kinetic entropy.

\section{Entropy of Complex Molecules}

As the example of water indicates, more complex molecular structures decrease the entropy compared to the ideal gas approximation. This is due to the interaction among the atoms of a molecule, as well as the interactions among the molecules in a given amount of material. Both these effects decrease the available phase space (e.g. compared to the ideal gas), and therefore these interactions also decrease the entropy. Especially the momentum space degrees of freedom are reduced as the interactions hinder the possibility of large momentum differences among the atoms of a molecule as well as among the molecules.

Furthermore, the atoms of a complex molecule may take different spatial configurations. This was not considered in the previous topological configuration estimates. The spatial configurations of the molecules depend also on the surroundings, so that a given molecule may have different configurations with different entropies. Especially in biology, these different configuration possibilities have important physiological roles. In case of molecules, these configurations are determined energetically, and can (in principle) be calculated based on the interaction energies within the molecule and among the molecules. Thus, the quantization of the phase space still enables us to obtain the absolute entropy of a given configuration. At a given temperature, one can also determine which configurations are realized, and with what probability. An example of such calculations is the calculation of protein and other molecule conformations, which is a widespread activity today.

The entropy of such a complex molecule is estimated indirectly via the Helmholtz free energy, $F$, where with the inverse temperature, $\beta=1 /\left(k_{B} T\right)$ we can calculate

$$
\beta F(T, V)=-S+E /\left(k_{B} T\right) .
$$

The effect of interaction is estimated via the correlation function of the density [6], by considering the atoms as hard spheres.

The same type of approximation is used in ref. [7], where the possible stable (or energy minimizing) packings were counted, and the Gibbs and Edwards (Boltzmann) entropy values were evaluated as a function of the system size. 
Obviously the number of possible configurations is much larger this way than in the previous topological estimates, but at the same time the locally energy minimizing configurations are considerably fewer than all configurations.

By counting the disordered 3D sphere packings this way the configuration entropy in the spatial occupation can be estimated. In the context of granular packings, our aim is to compute the number of ways, $\Omega$, in which $N$ spheres can be arranged in a given abstract volume of dimension $d$. Then the total available abstract volume in $d N$-dimensional space is $v$. We can consider the volume of the basis of attraction of each atom in the molecule, in a distinct energy minimum

$$
\mathcal{V}=\sum_{i=1}^{\Omega} v_{i}
$$

where $v_{i}$ is the volume of the $i$-th basin of attraction, $\Omega$ is the total number of distinct minima and $v$ is the accessible volume. Thus,

$$
\Omega=\frac{v}{\langle v\rangle},
$$

where $\langle v\rangle$ is the mean basin volume. Then the (Gibbs) entropy can be obtained as

$$
S_{G}=-\sum_{i=1}^{\Omega} p_{i} \ln p_{i}-\ln (N !),
$$

where $p_{i}=v_{i} / v$. A basic estimate is that the dimensionless entropy is

$$
S_{G} \approx N / 2
$$

for molecules of up to $\mathrm{N}=100$ atoms [7]. Then the entropy increases slower. So, we estimate that for very large molecules the specific spatial configuration entropy increases slower, e.g. as $\propto \ln N$.

In case of a given bio-molecule, like the DNA, not all spatial configurations are realized. Consequently the sum in eq. (19) is reduced to a single configuration or to a few configurations, which results in a specific entropy of the order

$$
S_{G}=-p_{i} \ln p_{i}-\ln (N !) \approx \frac{\langle v\rangle}{v} \ln \left(\frac{\langle v\rangle}{v}\right),
$$

and since $v=N \cdot\langle v\rangle$

$$
\sigma_{G} \approx \frac{1}{N} \ln (N)
$$

Therefore, in the specific entropy per $1 \mathrm{~kg}$ or per atomic number, the contribution is of the order of one and decreasing with increasing number of atoms, $N=N_{a}$, in the molecule.

As in most of the literature, these approaches give an entropy value which serves well for the comparison of different complex molecular configurations. At the same time, an absolute entropy value for a given complex molecule and for a given spatial configuration is not given, although this could in principle be possible.

The physical entropy of a complex molecule can be calculated based on all degrees of freedom in the phase space, and all interactions among the constituent atoms. In real situations, to calculate all possible realized and not realized configurations with their energies, is beyond our possibilities. So many different approaches were constructed based on the interconnected network of the constituents [8]. This way static entropies may characterize the global disorder of network topology. One of the approaches to define network entropy is based on the number of the neighbors of a node. This is a degree-based entropy, meaning it is low when the degree of the nodes is uniform and small. There exists more complex definitions of entropy, which account for the interaction between all possible pairs of nodes. The analysis of the entropy of representative subnetworks has also become possible.

Large molecules can form polymers, or even larger macromolecules, which are composed of many repeated subunits. These may have a broad range of properties in the case of both synthetic and natural polymers. In these cases the previous dense packing approach is not applicable. 


\section{DNA in Bacteria}

The level of complexity is increased in live structures that can replicate themselves. The basis of this replication is the deoxyribonucleic acid (DNA) molecule, which structure is a double spiral. The DNA stores a code made of four chemical bases: adenine (A), guanine $(\mathrm{G})$, cytosine $(\mathrm{C})$ and thymine $(\mathrm{T})$. The sequence of these bases determines the information available for building and maintaining an organism. DNA bases pair up with each other, A with T and $\mathrm{C}$ with $\mathrm{G}$, to form units called base pairs. Each base is also attached to other molecular structures which together, the base and its support, is called a nucleotide. The DNA can replicate, or make copies of itself. Bacteria, as asexual organisms, inherit identical copies of their parent's genes.

The average weight of a DNA base pair (bp) is 650 daltons or $650 \mathrm{AMU}$ (where $1 \mathrm{AMU}=1.660539040(20) \cdot 10^{-27}$ $\mathrm{kg}=931.4940954(57) \mathrm{MeV} / \mathrm{c}^{2}$.)

One of the smallest live systems is the endosymbiotic $2^{2}$ bacteria Candidatus Carsonella ruddii $(\mathrm{CCr})$, which has $N=159662$ base pairs. Its genome is built up by a circular chromosome.

The possible number of configurations of this number of base pair sequences is $4^{N}$, so the probability of the single existing $\mathrm{CCr}$ sequence is $p_{i}=4^{-N}$. The corresponding base pair sequence entropy is then

$$
\sigma_{P}^{b p}=H(X)=-p_{i} \ln p_{i}=-4^{-N} \ln 4^{-N}=N 4^{-N} \ln 4=1.386 N 4^{-N},
$$

thus

$$
\ln H(x)=-(1.386 N)+\ln N+\ln 1.386=-221291.532+11.981+0.326=-221279.225 \approx-2.2 \cdot 10^{5} .
$$

Thus, the specific entropy of the CCr DNA molecule on the base pair sequence configuration is

$$
\sigma_{P}^{b p} \approx e^{-221279}=10^{-96099}
$$

The DNA of the CCr is 159662 base-pairs and its weight is $m_{D N A_{C C r}}=159662 \cdot 650 \mathrm{AMU}=7.727 \cdot 10^{-18} \mathrm{~kg}$. So, the number of CCr DNA molecules in $1 \mathrm{~kg}$ is $N_{D N A_{C C r}}=1 / m_{D N A_{C C r}}=1.294 \cdot 10^{17}$.

The entropy of $1 \mathrm{~kg}$ of the DNA molecules based on their b.p. configuration is then

$$
S_{1 k g-C C r}^{b p}=k_{B} N_{D N A_{C C r}} \sigma_{P}^{b p}=1.786 \cdot 10^{-96105} \mathrm{~J} / \mathrm{K} .
$$

This DNA in the CCr bacteria builds up circular chromosomes. In the physical phase space the momentum part is largely negligible but the spatial configuration is substantial. For an exactly given sequence of length, in this case 159662 base pairs, the number of possible spatial configurations is large. It builds a circular chromosome, but even if the sequence is fixed, the shape can be different. For cell replication one might need a more straight configuration, and other more compact configurations are also possible although these might be energetically less favourable. Here we cannot estimate energetically all possible configurations and their probability. Instead, we make an overly simplified estimate.

The stretched out DNA could expand a sphere of radius $R=N / 2$ with a volume of $V=4 \pi R^{3} / 3=V=\pi N^{3} / 6=$ $8.673 \cdot 10^{30}$. If we position the 1 st base-pair to a certain location, the next base pair can be put to $\sim 25$ neighbouring points (if these are not occupied). As the volume is 25 orders of magnitude larger than the number of base-pairs, we can neglect the possibility that a chosen point is already occupied. Thus the estimated number of all connected spatial configurations can be

$$
M=25^{N}
$$

Here we assumed that the base-pairs can be connected in any angle within a cubic grid.

The number of circular configurations is much smaller. The circle has a circumference of $\mathrm{N}$ and the diameter of the circle is $D=N / \pi$. Let us choose a random point on the circle. Next, let us choose the opposite point, which is on a sphere of $4 \pi D^{2}$. These two points give an axis of the circle. The third point should set the plane of the circle, which

\footnotetext{
${ }^{2}$ Endosymbiont is an organism that lives within the body or cells of another organism.
} 
is on a circle of length $D \pi$. After we have chosen these three points, the circle is fixed and there is no more freedom. So the number of possible circles is

$$
M_{c}=4 \pi D^{2} \times D \pi=4 \pi^{2} D^{3}=4 N^{3} / \pi .
$$

Thus the probability of an arbitrary spatial configuration is $p_{i}=1 / M$, and the spatial configuration (s.conf.) entropy is

$$
\sigma_{P}^{\text {s.conf. }}=H(X)=-\sum_{i=1}^{M c} p_{i} \ln p_{i}=\frac{M_{c}}{M} \ln M=\frac{4 N^{3}}{\pi 25^{N}} \ln 25^{N}=\frac{4 N^{3}}{\pi 25^{N}} N \ln 25=\frac{43.219}{\pi} 25^{-N} N^{4}=4.099 N^{4} 25^{-N}
$$

thus

$$
\ln H(X)=1.411+4 * \ln N-N \ln 25=-513902.644 \approx-5.139 \cdot 10^{5} .
$$

Thus, the specific entropy of the CCr DNA molecule on the spatial configuration is

$$
\sigma_{P}^{\text {s.conf. }} \approx e^{-513903}=10^{-223183}
$$

This is a similarly low specific entropy as the one from the base-pair sequence, eqs. 23/24]. The entropies from these two independent degrees of freedom should be added

$$
\sigma_{P}=\sigma_{P}^{b p}+\sigma_{P}^{\text {s.conf. }},
$$

where actually the larger $\sigma_{P}^{b p}$ dominates, so that

$$
\sigma_{P}^{D N A} \approx 10^{-96099},
$$

The entropy of the DNA molecules in $1 \mathrm{~kg}$ matter is then

$$
S_{1 k g-C C r}^{D N A}=k_{B} N_{D N A C C r} \sigma_{P}^{D N A}=1.786 \cdot 10^{-96105} \mathrm{~J} / \mathrm{K} .
$$

This is the same as the entropy of the DNA b.p. configuration only, eq. 26], because the entropy from the spatial configuration is utterly negligible.

If we would count the spatial configuration only that would give a smaller specific entropy value, and the resulting entropy for the DNA molecules would yield a smaller value

$$
S_{1 k g-C C r}^{\text {s.conf. }}=k_{B} N_{D N A_{C C r}} \sigma_{P}^{D N A}=1.786 \cdot 10^{-223191} \mathrm{~J} / \mathrm{K} .
$$

\section{Entropy of the CCr bacterium}

Without its environment the DNA molecule cannot exist. If we want to calculate the entropy of one kg material we have to estimate the other surrounding constituents of in the CCr bacteria, in comparison with the DNA in the cell.

The total weight of a $\mathrm{CCr}$ bacterium can be estimated to $m_{C C R} \approx 2.3 \cdot 10^{-17} \mathrm{~kg}$, i.e. about three times bigger than the weight of the DNA, so two thirds of this weight is made up of smaller molecules than the DNA. The number of CCr bacteria in $1 \mathrm{~kg}$ is $N_{P}=m_{C C R}^{-1}=0.4 \cdot 10^{17} / \mathrm{kg}$. Two thirds of this matter is approximated to be water, and one third of the weights is given by the DNA molecules.

The number, and thus the entropy, of the DNAs in $1 \mathrm{~kg} \mathrm{CCr}$ bacteria is then about three times less than estimated in eq. 49,

$$
S_{1 k g-C C r}^{D N A}=k_{B} N_{P} \sigma_{P}^{D N A}=0.595 \cdot 10^{-96105} \mathrm{~J} / \mathrm{K}
$$

For a first, extremely rough estimate, we can assume that this extra material has about the same entropy as water, $S_{1 \mathrm{~kg}}^{\mathrm{H}_{2} \mathrm{O}} \approx 4000 \mathrm{~J} / \mathrm{K}$. If we then calculate the entropy of $1 \mathrm{~kg} \mathrm{CCr}$ bacteria:

$$
S_{1 k g}^{C C r}=\frac{2}{3} S_{1 k g}^{H_{2} O}+S_{1 k g}^{D N A}=2666 \mathrm{~J} / \mathrm{K}+0.595 \cdot 10^{-96105} \mathrm{~J} / \mathrm{K} .
$$


Thus, the contribution of the DNA would be utterly negligible to the total entropy of $1 \mathrm{~kg} \mathrm{CCr}$ bacteria. The replacement of smaller molecules by water overestimates the entropy unrealistically.

A more realistic estimate is that the DNA leads to the build up of different molecules in the cell. Their number and variation depends on the DNA, but also on the environment (!). Due to the larger number and weight of these other molecules of large variation and complexity, the possible number of variations increases, compared to the possible variations of the DNA structure. At the same time, the number of realized configurations can be an even smaller proportion, due to the additional selection caused by the environmental conditions. In conclusion, we can estimate that the complexity of all the smaller molecules of the cell is larger, and their entropy is smaller, than the one estimated from the complexity of the DNA molecule itself.

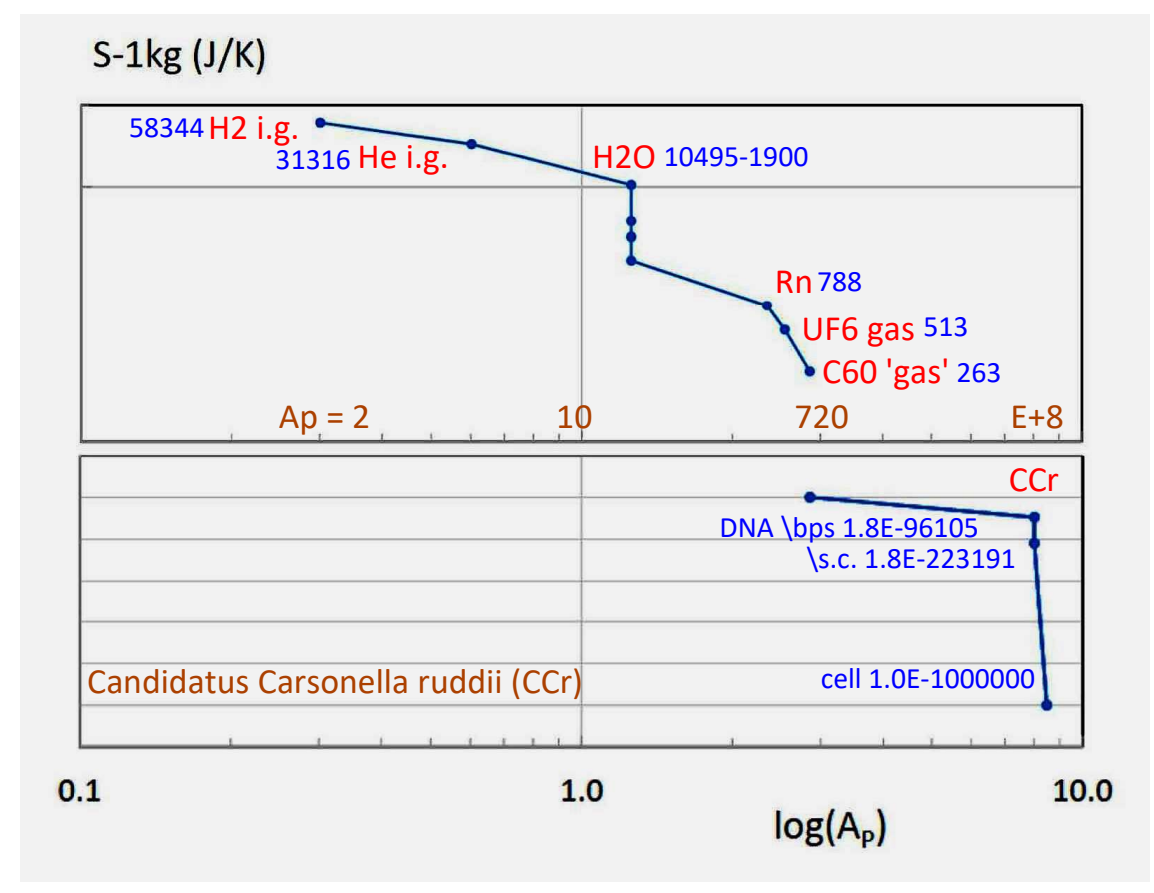

Figure 7. (color online) The entropy of $1 \mathrm{~kg}$ material, $S_{1 \mathrm{~kg}}$ in units of $(\mathrm{J} / \mathrm{K})$ from light molecules behaving like ideal gases up to the DNA of the simplest bacterium, Candidatus Carsonella ruddii. This last one is based on its base-pair sequence (bps), its spatial configuration (s.c.), and all other different molecules inside the cell. The entropy values are plotted versus the atomic number of the molecules, which is the weight of the molecule in daltons.

Based on this reasoning, for unit mass, the entropy of all the smaller molecules may be even smaller than the entropy of the DNA, so the more realistic estimate for the entropy of $1 \mathrm{~kg} \mathrm{CCr}$ bacteria is

$$
S_{1 k g}^{C C r}=k_{B} N_{P} \sigma_{P}^{D N A}=1.0 \cdot 10^{-1000000} \mathrm{~J} / \mathrm{K}
$$

It is still amazing to estimate and see, how extremely small the entropy of the most complex molecules and structures is.

\section{Entropy of Highly Complex Molecules}

The building blocks of DNA are the nucleotides, Thymine, Adenine, Guanine and Cytosine. Each Thymine connects to an Adenine and each Guanine connects to a Cytosine. This is one allele. Such a pair is called a nucleotide base pair.

The number of DNA base pairs (DNA-bp) in the total human genome (in the 23 chromosomes) is approximately 3.2 billion $\left(A_{P}^{D N A-b p}=3.234 \cdot 10^{9}\right)$. Each chromosome is a curled up DNA molecule. In these DNAs there are some 
20000-25000 genes that contain these base pairs. Genes are sections of the DNA which act as instructions to make molecules, e.g. proteins. Genes with a smaller amount of base pairs (smaller DNA section) will have a higher entropy per mass while a longer DNA section will decrease the entropy.

The human body contains approximately 10 trillion $\left(10^{13}\right)$ cells and each of those typical cells contain the genome, i.e. 23 chromosome pairs. There is then approximately $6.4 \cdot 10^{22}$ base pairs in total, but since the genome is the same in each cell, the cell count does not increase the sequential configuration entropy from DNA.

The total amount of all sequences is $N_{\text {all-sequences }}=4^{N}$, where $N$ is the number of base pairs,

$$
N_{b p}=A_{P}^{D N A-b p} .
$$

Assuming that all sequences are of equal probability, then for a given sequence, $k$, the probability is $p_{k}=1 / 4^{N_{b p}}$ and we get

$$
H_{\text {max }}=\ln 4^{N_{b p}}=N_{b p} \ln (4)=4.482 \cdot 10^{9}
$$

by adding up all these probabilities for $k=1, \ldots, 4^{N_{b p}}$. This is the Shannon entropy for an ensemble where all possible DNA sequences were realized. If all humans would have the same unique DNA sequence then the Shannon entropy of a human and of the identical species would be

$$
\sigma_{P}^{b p}=H(X)=-p_{i} \ln p_{i}=1 / 4^{N_{b p}} \cdot \ln 4^{N_{b p}}=N_{b p} \cdot \ln 4 / 4^{N_{b p}}=1.386 N_{b p} / 4^{N_{b p}}
$$

and thus for a single sequence without any variation in the species:

$$
\ln H(X)=\ln \left(4.483 \cdot 10^{9}\right)-N_{b p} \ln 4=-4.483 \cdot 10^{9}
$$

\section{Single Nucleotide Polymorphisms}

Every human is genetically different, and one of the most common genetic variations is called Single Nucleotide Polymorphisms (SNP). An SNP could be the replacement of Cytosine with Adenine for example. The two possible variations are two alleles for this base position.

Let us now estimate the number of different sequences in the whole human genome (i.e. in the total of 23 chromosomes or 23 DNAs. One person has only one DNA sequence in each of his or her cells (except mutations developed due to external influences in life). On the other hand different individuals in the species may have variations in their DNA sequences. These genetic variations are the Single Nucleotide Polymorphisms (SNP). This happens on average once for 300 locations, and mostly only one type of exchange is possible, i.e. one allele.

Also, if we do not consider any other variation, the Shannon entropy can be calculated from eq. (3). We assume that the "standard" Human genome sequence is known.

We want to sum up the argument of the Shannon entropy expression, $p_{i} \ln p_{i}$, over all base pair configurations. We use the relation for the summation

$$
\sum_{i=0}^{2^{N_{b p}}} \rightarrow \sum_{i=0}^{N_{b p}} \frac{N_{b p} !}{\left(N_{b p}-i\right) ! i !}
$$

Shannon entropy for a sequence with $N_{b p}$ base pairs and $i$ SNP's is then:

$$
H(X)=-\sum_{i=0}^{N_{b p}} \frac{N_{b p} !}{\left(N_{b p}-i\right) ! i !} P_{A}^{i}\left(1-P_{A}\right)^{N_{b p}-i} \ln \left[P_{A}^{i}\left(1-P_{A}\right)^{N_{b p}-i}\right],
$$

where $P_{A}=1 / 300$. This counts up the number of possible SNP changes that may happen.

Notice that [7]:

$$
\sum_{i=0}^{N_{b p}} \frac{N_{b p} !}{\left(N_{b p}-i\right) ! i !} P_{A}^{i}\left(1-P_{A}\right)^{N_{b p}-i}=1 \text {. }
$$

The function ln can be rewritten

$$
\ln \left[P_{A}^{i}\left(1-P_{A}\right)^{N_{b p}-i}\right]=N_{b p} \ln \left[1-P_{A}\right]+i \ln \left[\frac{P_{A}}{1-P_{A}}\right] .
$$


Putting this in the above equation we get

$$
H(X)=-N_{b p} \ln \left[1-P_{A}\right]-\ln \left[\frac{P_{A}}{1-P_{A}}\right] \times \sum_{i=0}^{N_{b p}}\left[\frac{N_{b p} ! i}{\left(N_{b p}-i\right) ! i !} P_{A}^{i}\left(1-P_{A}\right)^{N_{b p}-i}\right] .
$$

Since the values of $i$ that contribute the most are around $N_{b p} \times P_{A}$ we use the approximation that the $i$ in the numerator is a constant instead of a variable.

$$
i \rightarrow k=N_{b p} P_{A}
$$

We can then approximate $H(x)$

$$
H(X) \approx-N_{b p} \ln \left[1-P_{A}\right]-N_{b p} P_{A} \ln \left[\frac{P_{A}}{1-P_{A}}\right]=N_{b p}\left[-P_{A} \ln \left[\frac{P_{A}}{1-P_{A}}\right]-\ln \left(1-P_{A}\right)\right] .
$$

We see that $H(X)$ is proportional to $N_{b p}$ and $P_{A}=1 / 300$ so that

$$
H_{\max }(X) \approx 0.0223 \cdot N_{b p}=7.15 \cdot 10^{7},
$$

for all possible SNP configurations of the Human species. This is much smaller than the entropy corresponding to the maximum possibility of all variations for the DNA of a length of the human genome.

The spatial configuration of the DNA can lead to even higher complexity than the base-pair sequence. The spatial area that could be reached by the DNA may reach $N_{b p}^{3}$, when the length is $N_{b p}$. Thus the probability for one configuration could be of the order of $N_{b p}^{-2}$. On the other hand, our knowledge on the spatial configuration of the DNA of the human genome is little, and the DNA exists in different configurations: curled up in chromosomes, or straightened out at cell division. Thus, to study the entropy arising from the spatial configuration of DNA would require knowledge that we do not have today.

Before cell division, the chromosomes are reshaped and the DNA takes a linearly extended shape (like an extended line), which enables more motion and the replication of the DNA. In this configuration the degrees of freedom in the physical phase space are increased, and thus the entropy of the configuration also increases. This extending of the DNA molecule requires additional energy temporarily. The linear shape allows more motion and increases the possibility of occupying a larger part of the phase space.

Thus the compact curled up configuration of DNA has smaller entropy, having similar amount of freedom as atoms in a solid state, while the linearly extended molecule has considerably larger entropy, similar to a liquid type of structure. So, the entropy of the DNA changes dynamically.

The average weight of a DNA can be obtained from its length, $N_{b p}=3.234 \cdot 10^{9}$, times the number of atoms per base-pair, 650. Thus $m_{D N A}=650 \cdot N_{b p} \mathrm{AMU}=3.489 \cdot 10^{-15} \mathrm{~kg}$. Consequently in $1 \mathrm{~kg}$ we have $N_{D N A}=2.866 \cdot 10^{14}$ molecules.

Then, from eq. (40) we get the specific entropy for the DNA base-pair sequence of a single individual. The corresponding entropy in $1 \mathrm{~kg}$ of DNA molecules is then

$$
S_{1 k g}^{D N A}=k_{B} N_{D N A} \sigma_{P}^{D N A}=3.958 \cdot 10^{-1947000000} \mathrm{~J} / \mathrm{K}
$$

\section{Entropy of Live Material Tissues}

Live material tissues contribute to a high complexity on a larger scale. The best example of this is the nervous system. In a human brain there are about $10^{10}$ nerve cells, and each of these have about $10^{3}-10^{5}$ synaptic junctions. This means that the whole nervous system may have up to $N_{s}=10^{15}$ synaptic junctions. If we quantize the synaptic couplings as 0 to 1 only, then we have $2^{N_{s}}$ different states statically. This can even lead to higher complexity than the DNAs. At the same time it is difficult to estimate, which configurations can be realized, and actually these configurations can change each second. A sleeping persons brain has different entropy than an active one.

To analyse this level of complexity is beyond our present goal. 


\section{Conclusions}

We have demonstrated quantitatively the increasing complexity of materials, and used the entropy for unit amount of material in order to be able to get a measure. This idea stems from Ervin Schrödinger, but our knowledge today makes it possible to extend the level of quantitative discussion to complex live materials.

We may continue these studies to higher levels of material structures, like living species, artificial constructions, symbiotic coexistence of different species, or groupings of the same species. We can even continue up to structures in Human society.

The main achievement of this work is to show how the entropy in the physical phase space and the entropy of structural degrees of freedom (Shannon entropy) can be discussed on the same platform, as shown in section 3 via eq. (14). For further developments it is important to point out two fundamental aspects of the entropy concept: (i) the quantization of the space of a given degree of freedom, and (ii) the selection of the realized, realizable or beneficial configurations from all the possible ones. If we go towards more complex systems, these two questions become nontrivial, and particularly in the case of the utmost complex systems where it is not clear which are the realizable and most beneficial systems. This is already a challenging question in the case of the nervous system, and even more so for organizations in the society or in economy.

The examples presented are all analyzed from a static point of view. As we see on the example of the nervous system the dynamical change of the entropy of the system is also important. Furthermore, the speed of development is also important. The early development of complexity happened on a very slow rate, while the development of complexity of the nervous system is many orders of magnitude more rapid then the development of the DNA structure. The dynamics and direction of these changes are also essential, as shown in ref. [3].

Some specific studies in these directions exist already. See e.g. considerations on general dynamics of sustainable development [9], and on the life-span of different species related to their entropy and metabolism. We are looking forward to further developments [3].

\section{Acknowledgements}

Enlightening discussions with Tamás Biró, Stuart Holland, Zoltán Néda, Gábor Palló, István Papp, Péter Ván and Yilong Xie are gratefully acknowledged. This research was partially supported by the Academia Europaea Knowledge Hub Bergen, by the Institute of Advanced Studies, Kőszeg, and by the Research Council of Norway, grant no. 231469.

\section{References}

[1] Erwin Schrödinger: What is life? - The Physical Aspect of the Living Cell, (The Cambridge University Press, 1944) Based on the Lectures delivered under the auspicies of the Trinity College, Dublin, in February 1943.

[2] Shannon, Claude E. . A Mathematical Theory of Communication. Bell System Technical Journal. 27, (1948) 379423; James V Stone: Information Theory: A Tutorial Introduction (Sebtel Press 2015); Mikhail V Volkenstein: Entropy and Information (Progress in Mathematical Physics, 2009); Robert M. Gray: Entropy and Information Theory (Sptinger 2014).

[3] L. Pénzes, and L.P. Csernai, Über den Zusammenhang von Lebensdauer, Konstitution und Information, Zeitschrift für Alternsforschung, 35, 285-296 (1980).

[4] L.P. Csernai, I. Papp, S.F. Spinnangr and Yilong Xie, Physical Basis of Sustainable Development, Journal of Central European Green Innovation, 4, 39-50 (2016).

[5] L.P. Csernai, Introduction to Relativistic Heavy Ion Collisions (Wiley, 1994).

[6] James F. Lutsko and Marc Bauss, Can the Thermodynamic Properties of a Solid Be Mapped onto Those of a Liquid?, Phys. Rev. Lett. 64, 761 (1990).

[7] S. Martiniani, K.J. Schrenk, J.D. Stevenson, D.J. Wales and D. Frenkel, Turning intractable counting into sampling: Computing the configurational entropy of three-dimensional jammed packings, Phys. Rev. E 93, 012906 (2016).

[8] Peter Csermely, Kuljeet Singh Sandhu, Eszter Hazai, Zsolt Hoksza, Huba J.M. Kiss, Federico Miozzo, Daniel V. Veres, Francesco Piazza and Ruth Nussinov, Disordered Proteins and Network Disorder in Network Descriptions of Protein Structure, Dynamics and Function: Hypotheses and a Comprehensive Review, Current Protein and Peptide Science, 13, 19-33 (2012).

[9] Tamas S. Biro, Zoltan Neda, Dynamical Stationarity as a Result of Sustained Random Growth arXiv:1611.06698 [cond-mat.stat-mech] 